\title{
Mechanical and Optical Behavior of Double Network Rubbers
}

\author{
P. H. Mott and C. M. Roland* \\ Chemistry Division, Code 6120, Naval Research Laboratory, Washington, D.C. 20375-5342 \\ Received J anuary 27, 2000
}

\begin{abstract}
Stress, strain, and optical birefringence were measured for a series of peroxide-cured natural rubbers, having both isotropic and double network structures. The residual stretch (permanent set) for the latter ranged from 2.0 to 4.5, with elastic moduli that were an increasing function of this residual strain, as found in previous works. A small birefringence, ca. $10^{-5}$, was observed for the unstressed double networks, and its magnitude increased with increasing residual strain. The sign of this birefringence corresponded to extension of the (undeformed) double networks. Under stress, the birefringence foll owed the stress optical law. The double network properties were interpreted using the constrained-chain model of rubber elasticity, with the assumption of independent, additive contributions from the two component networks. The calculated results differed from the experimental findings, in particular underestimating the residual strain. This failure is a consequence of the overprediction of the stresses during compression, a limitation common to molecular theories of rubber elasticity. The modeling of the double networks does account qualitatively for the sign of their unstressed birefringence, which is due to the stressoptical coefficient being larger in tension than in compression. This particular deviation from the stressoptical law is known from both theory and experiment.
\end{abstract}

\section{Introduction}

The properties of an elastomeric network depend not only on the density of junctions (cross-links) but also on the distribution and orientation of the chains when the junctions are formed. The importance of chain orientation is emphasized in double network rubbers, which are elastomers cross-linked twice, the second time while the material is deformed. Many studies have been carried out on double networks, ${ }^{1-8}$ including their use to evaluate the contribution of trapped entanglements to rubber el asticity. ${ }^{9-14}$ Double networks can also arise spontaneously via chain scission, ${ }^{15,16}$ via strain-induced crystallization, ${ }^{17-19}$ or in the presence of reinforcing fillers. ${ }^{20,21}$

Theoretical interpretations of these rubbers are based on the idea that the component networks behave independently, so that the mechanical response is the sum of the individual contributions. ${ }^{3,22-24}$ The strain energy for uniaxial deformation of a double network is thus given by

$$
W_{1-2}(\lambda)=W_{1}(\lambda)+W_{2}\left(\lambda / \lambda_{x}\right)
$$

where the subscripts 1 and 2 refer to the first and second networks, respectively. The stretch ratio of the composite network, $\lambda$, is referenced to the original, undeformed first network, and $\lambda_{x}$ is the stretch during the second cross-linking; hence, $\lambda=\lambda_{1}$ and $\lambda_{2}=\lambda / \lambda x$. The permanent set of the final material, defined as the unstressed stretch ratio $\lambda_{R}$, reflects the balance of forces between the two networks. Expressed in terms of the engineering stresses $\sigma$ of the component networks,

$$
\sigma_{1}\left(\lambda_{R}\right)=-\sigma_{2}\left(\lambda_{R} / \lambda_{X}\right)
$$

For the usual circumstance in which the first network is in tension during the second cure, $\lambda_{R}<\lambda_{x}$.

Double networks can be viewed as interpenetrating polymer networks in which the same chain segments belong to both networks and, more importantly, the component networks are oriented. It is this orientation that gives rise to enhancement $5,7,8$ and anisotropy 25 of the mechanical properties. The expectation, borne out by experiment,, $25-28$ is that the modulus of a double network rubber will differ from the modulus of the corresponding isotropic elastomer; at higher residual strains, the equilibrium modulus is higher.

Unstressed double networks have been shown to be birefringent. ${ }^{28}$ This is at odds with the stress optical law, ${ }^{29}$ which for uniaxial deformation is

$$
\Delta \mathrm{n}=\mathrm{C} \lambda \sigma
$$

where $\Delta \mathrm{n}$ is the birefringence, C the stress-optical coefficient, and $\lambda \sigma$ the true stress. Although this unstressed birefringence has been observed, ${ }^{28}$ it was not systematically explored, and no analysis was attempted.

The purpose of the present work was to examine the behavior of double networks using molecular theories of rubber elasticity, including quantifying their anisotropy using optical bir refringence. As has been shown, ${ }^{30-32}$ molecular elasticity models cannot describe the full range of deformation behavior of rubbery networks, as made apparent when tension and compression data are considered simultaneously. This is the situation prevailing with double networks; therefore, their study can provide insight into the general problem of the elasticity of high polymers.

\section{Experimental Section}

The polymer was cis-1,4-polyisoprene, in the form of deproteinized natural rubber $(\mathrm{H}$. A. Astlett Co.), with $2.0 \mathrm{phr}$ dicumyl peroxide (Varox DCP-R, from R.T. Vanderbilt Co.) added using a two-roll mill. The first networks, designated SA, $\mathrm{SB}$, or SC, were cured in a compression mold at $125 \mathrm{C}$, for $40-140 \mathrm{~min}$, reacting from $15 \%$ to $39 \%$ of the peroxide (see Table 1). To produce the double networks listed in Table 2, the rubber was then stretched using a frame, with the extension ratio measured by fiducial marks. The second cure was carried out in a vacuum chamber, while the stretched samples were contained between two aluminum plates, with heat applied from both sides, using independently controlled heaters. The assembly reached temperature $\left(160^{\circ} \mathrm{C}\right)$ in about 
Table 1. First (Precursor) Networks

\begin{tabular}{ccc}
\hline first network & Cure time $\left(\min\right.$ at $\left.125^{\circ} \mathrm{C}\right)$ & $\mu_{1}\left(\mathrm{~mol} / \mathrm{m}^{3}\right)$ \\
\hline SA & 40 & 14.9 \\
SB & 105 & 32.0 \\
SC & 140 & 39.3
\end{tabular}

Table 2. Experimental Results for Double Networks

\begin{tabular}{cccccc}
\hline $\begin{array}{c}\text { precursor } \\
\text { network }\end{array}$ & $\begin{array}{c}\text { double } \\
\text { network }^{\mathrm{a}}\end{array}$ & $\lambda_{\mathrm{x}}$ & $\lambda_{\mathrm{R}}$ & $\begin{array}{c}\text { modulus } \\
(\mathrm{kPa})^{\mathrm{b}}\end{array}$ & $\Delta \mathrm{n}\left(\times 10^{6}\right)$ \\
\hline SA & DA0 & 1 & 1 & 687 & 0 \\
SA & DA1 & 2.86 & 2.52 & 715 & 5.4 \\
SA & DA2 & 3.95 & 3.30 & 757 & 7.4 \\
SA & DA3 & 4.93 & 4.13 & 860 & 9.1 \\
SA & DA4 & 5.76 & 4.50 & 1040 & 11.8 \\
SB & DB0 & 1 & 1 & 687 & 0 \\
SB & DB1 & 2.84 & 2.26 & C & 9.3 \\
SB & DB2 & 3.88 & 2.72 & C & 16.8 \\
SC & DC0 & 1 & 1 & 687 & 0 \\
SC & DC1 & 2.75 & 2.03 & C & 16.0
\end{tabular}

a $\mu_{1-2}=\mu_{1}+\mu_{2}=102 \mathrm{~mol} / \mathrm{m}^{3}$ for all double networks. ${ }^{\mathrm{b}}$ Stress at $100 \%$ strain. ${ }^{c}$ Not measured.

$20 \mathrm{~min}$ and was maintained there for $10 \mathrm{~min}$, followed by quenching. This consumed the remaining peroxide, with negligible degradation of the first network. The isotropic, twicecured networks (i.e., DA0, DB0, and DC0, for which $\lambda_{x}=1$ ) were verified to have equal moduli. Any nonuniform heating or oxidative degradation gave rise to stresses near the edges. Although the procedure minimized these problems, $15 \mathrm{~mm}$ was trimmed from the edge of each specimen. The resulting 12.5 $\mathrm{mm}$ wide strips had uniform birefringence and remained flat (no curling) when removed from the frame.

All measurements were done at room temperature. Uniaxial extensi on measurements used an Instron 4206 with a Wallace optical extensometer (elongation rate of $3.33 \times 10^{-4} \mathrm{~s}^{-1}$, which is $2 \% / \mathrm{min})$. Mechanical equilibrium data were obtained by dead-weighting and measuring the displacement with a cathe tometer.

For the birefringence data, the light retardation was measured with a Sénarmont compensator, ${ }^{33}$ which involves a pair of polarizing filters and a quarter-wave plate. The birefringence is determined by

$$
\Delta \mathrm{n}=\frac{\theta \Lambda}{\pi \mathrm{l}}
$$

where $\theta$ is the rotation angle of the polarizing filter necessary to extinguish the transmitted intensity, $\Lambda$ is the wavel ength of the light (=632.8 $\mathrm{nm}$ for HeNe laser), and I, the optical path length, is the sample thickness. This method provides the sign of the birefringence, which reveals whether the apparent orientation corresponds to tension or compression.

\section{Results}

Uniaxial extension data were obtained on all networks, with the effect of relaxation corrected using the factorable Kaye-BKZ equation. ${ }^{34,35}$ The engineering stress is thus

$$
\sigma(t)=\int_{0}^{t} E(t-u) g(\lambda) \frac{\partial \lambda(u)}{\partial u} d u
$$

where $\mathrm{E}(\mathrm{t})$ is the stress relaxation modulus, and the damping function, $g(\lambda)$, describes the strain dependence of the equilibrium modulus. By assuming the effects of time and strain to be separable, departures from strict linear viscoelasticity are circumvented. The validity of eq 5 has been demonstrated for cross-linked rubber under large deformation, provided no strain reversal occurs. ${ }^{36,37}$ F or the twice-cured networks, stress relax-

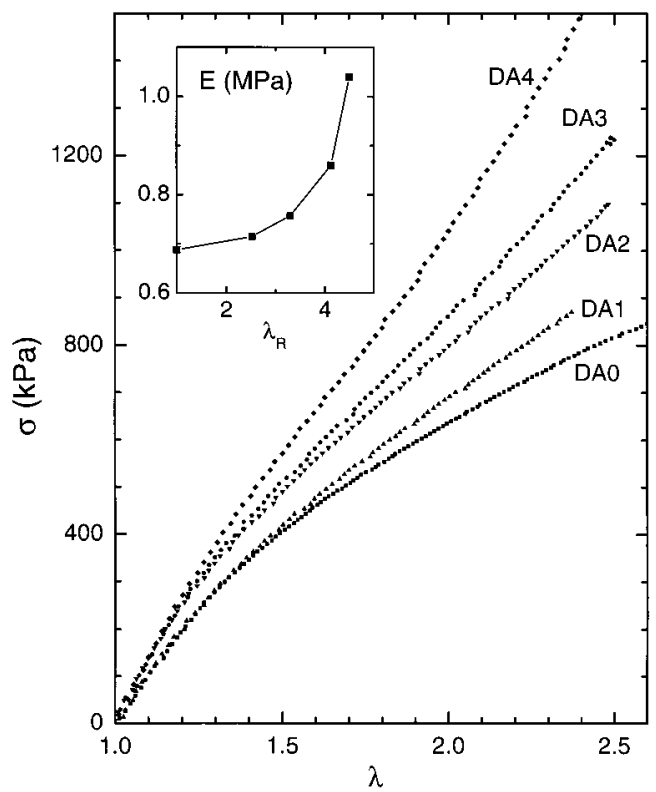

Figure 1. Engineering stress as a function of stretch ratio for the DA double networks, prepared from the single network precursor SA (Table 1). The inset shows the stress at $100 \%$ strain for each of these double networks, as identified by their residual strain (see Table 2 ).

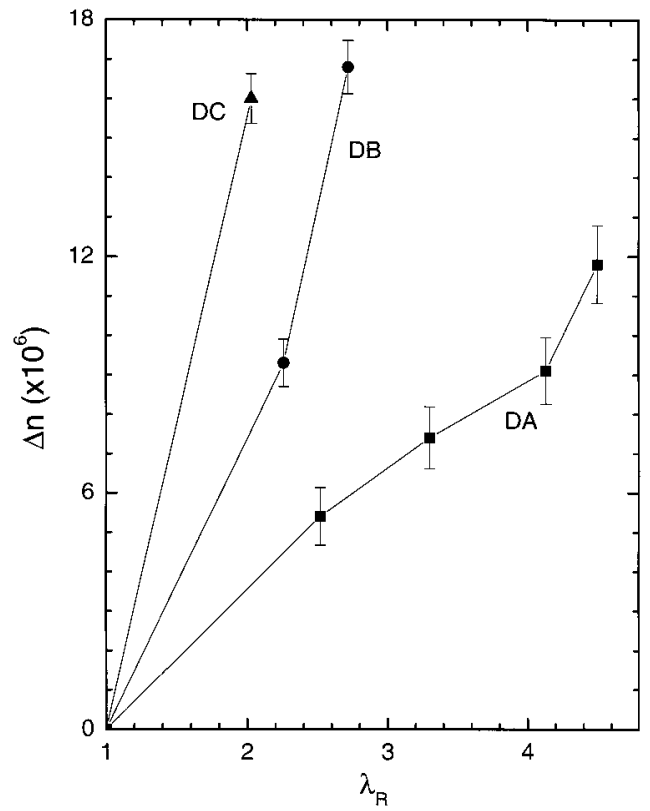

Figure 2. Birefringence measured at zero stress for the double networks, as a function of their residual stretch.

ation was minimal; thus, the correction from eq 5 was important only for the (partially cured) single networks.

Shown in Figure 1 are equilibrium stress-strain curves for the double networks referred to as DA, formed from the SA precursor network. The mechanical behavior varies systematically with residual strain, as shown previously. ${ }^{5,25-28}$ The stresses of the double network rubbers exceed that of the corresponding isotropic network (i.e., a network having equal cross-link density with $\left.\lambda_{x}=1\right)$. This is further illustrated in the inset to Figure 1, in which the secant modulus (stress at $100 \%$ strain) is shown to increase with increasing residual strain.

In Figure 2 we display the birefringence measured for all the double networks at zero stress. F or a given 


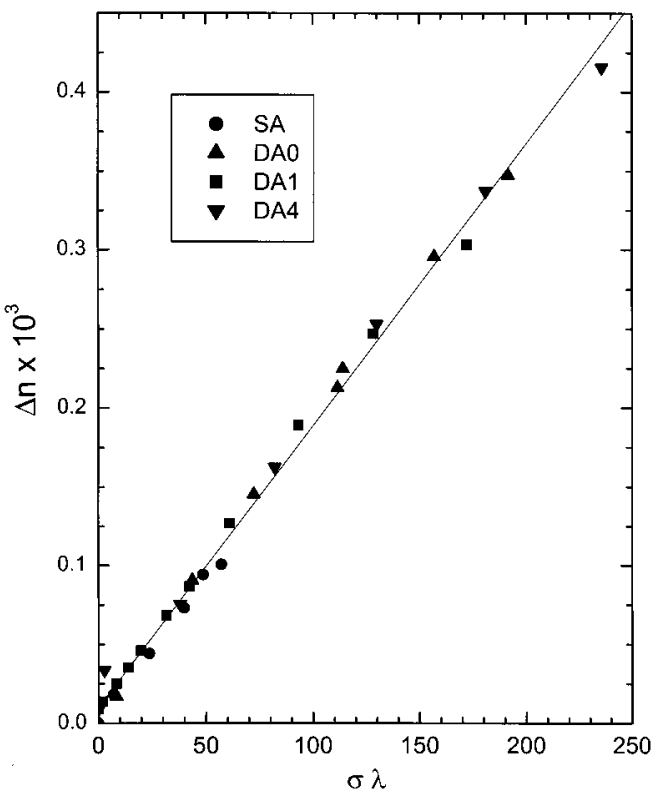

Figure 3. Birefringence as a function of true stress for various networks. The slope yields the stress optical coefficient, $\mathrm{C}=$ 1.8 $\mathrm{GPa}^{-1}$, which is independent of network structure.

precursor single network, there is an increase in $\Delta \mathrm{n}$ with increasing residual strain. On the other hand, increasing the proportion of cross-links in the first network (e.g., DA1 to DB1 to DC1, prepared at the same $\left.\lambda_{x}\right)$ increases the birefringence, even though the residual stretch $\lambda_{R}$ decreases. We also note that the sign of the birefringence, which has not previously been determined for double networks, corresponds to tension. This may seem obvious, since the residual strains are all el ongation; however, as discussed below, the sign of $\Delta \mathrm{n}$ at zero stress is intimately related to the strain dependence of the stress-optical coefficient.

The birefringence was also measured for the networks under tension. As seen in Figure 3, this stress birefringence is proportional to the true stress, consistent with eq 3. The slope of the line yields the stress optical coefficient, $\mathrm{C}=1.8 \mathrm{GPa}^{-1}$, a value in accord with previous determinations on natural rubber. ${ }^{32,38,39}$ The small intercept in Figure 3 represents the birefringence shown in Figure 2.

\section{Discussion}

Mechanical Response. Modeling the mechanical response of the double networks via eqs 1 and 2 requires accounting for the elastic behavior of each component network. Although we can measure the stress-strain curves of the first network, this cannot be done directly for the second network. Instead, the results for the unoriented $\left(\lambda_{x}=1\right)$ double network must be used to deduce this information.

To carry out this analysis, we employ the diffused contraint (DC) model of rubber elasticity. ${ }^{40}$ The advantage of this approach is that the fitting parameters have molecular significance. There are other constitutive equations for rubber elasticity, $41-44$ including the phenomenological chain models, ${ }^{4-49}$ but these lack the physical insight provided by the constraint models.

In the spirit of the work of Flory et al., ${ }^{50-52}$ who pioneered this approach to describing rubber elasticity, the DC model posits two contributions to the stress, arising from cross-link junctions and from topological constraints. The latter comprise entanglements and excluded-volume interactions, which are assumed to act continuously along the chain. In the absence of diluent, the engineering stress $\sigma_{\mathrm{DC}}$ for the diffused constraint model is given by the sum of the stress for a phantom network, $\sigma_{\mathrm{ph}}$, which depends only on the cross-link density, and the stress due to the constraints ${ }^{40}$

$$
\sigma_{\mathrm{DC}}=\sigma_{\mathrm{ph}}\left[1+2 \int_{0}^{1} \frac{\lambda \mathrm{K}\left(\lambda^{2}\right)-\lambda^{-2} \mathrm{~K}\left(\lambda_{\perp}{ }^{2}\right)}{\lambda-\lambda^{-2}} \mathrm{~d} \Theta\right]
$$

where $\lambda_{\perp}$ is the stretch ratio perpendicular to the applied load $\left(=\lambda^{-1 / 2}\right.$ for uniaxial extension of an incompressible rubber), and $\Theta$ signifies the relative position along the network chain ( $\Theta=0$ and 1 at the network junctions). A phantom network, which experiences no topological interactions, has an engineering stress given by

$$
\sigma_{\mathrm{ph}}=\mu \mathrm{RT}\left(\lambda-\lambda^{-2}\right)
$$

in which $\mu$ is the cross-link density and RT has its usual significance. Both eqs 6 and 7 ignore chain ends, which are negligible for the high molecular weight natural rubber used herein. (More generally, the cross-link density is replaced by the cycle rank per unit volume. ${ }^{50}$ ) We also assume tetrafunctional junctions, as expected for peroxide cross-linked 1,4-pol yisoprene. ${ }^{53}$

The function $K\left(\lambda^{2}\right)$ in eq 6 is defined by

$$
\mathrm{K}\left(\lambda^{2}\right)=\frac{\mathrm{B}\left(\lambda^{2}\right) \dot{\mathrm{B}}\left(\lambda^{2}\right)}{\mathrm{B}\left(\lambda^{2}\right)+1}+\frac{\mathrm{D}\left(\lambda^{2}\right) \dot{\mathrm{D}}\left(\lambda^{2}\right)}{\mathrm{D}\left(\lambda^{2}\right)+1}
$$

where

$$
\begin{gathered}
\mathrm{B}\left(\lambda^{2}\right)=\frac{\kappa^{2}(\Theta)\left(\lambda^{2}-1\right)}{\left(\lambda^{2}+\kappa(\Theta)\right)^{2}} \\
\dot{\mathrm{B}}\left(\lambda^{2}\right) \equiv \frac{\partial \mathrm{B}\left(\lambda^{2}\right)}{\partial \lambda^{2}}=\mathrm{B}\left(\lambda^{2}\right)\left[\frac{1}{\lambda^{2}-1}-\frac{2}{\lambda^{2}+\kappa(\Theta)}\right] \\
\mathrm{D}\left(\lambda^{2}\right)=\frac{\lambda^{2} \mathrm{~B}\left(\lambda^{2}\right)}{\kappa(\Theta)}
\end{gathered}
$$

and

$$
\dot{\mathrm{D}}\left(\lambda^{2}\right) \equiv \frac{\partial \mathrm{D}\left(\lambda^{2}\right)}{\partial \lambda^{2}}=\frac{\lambda^{2} \dot{\mathrm{B}}\left(\lambda^{2}\right)+\mathrm{B}\left(\lambda^{2}\right)}{\kappa(\Theta)}
$$

Entanglements suppress fluctuations (Brownian motion) of the network segments. The degree of this suppression varies along the chain as

$$
\kappa(\Theta)=\bar{\kappa}\left[1+{ }^{4} / 3 \Theta(1-\Theta)\right]
$$

where $\bar{\kappa}$ is a constant, equal to 0 and $\infty$ for the limiting cases of phantom and affine behavior, respectively. ${ }^{50}$ Equation 6 assumes the constraint effect is distributed uniformly along the chain, although more complicated situations are possible. ${ }^{40}$

Representative equilibrium stress-strain measure ments are shown in Figure 4, for the single network SA and the isotropic double network DA0, plotted in the Mooney form of reduced stress, $\sigma /\left(\lambda-\lambda^{-2}\right)$, versus $\lambda^{-1}$. We have included previously obtained tension and compression results on a rubber identical to DA0, referred to in the prior publication as NR2. ${ }^{32}$ There is 


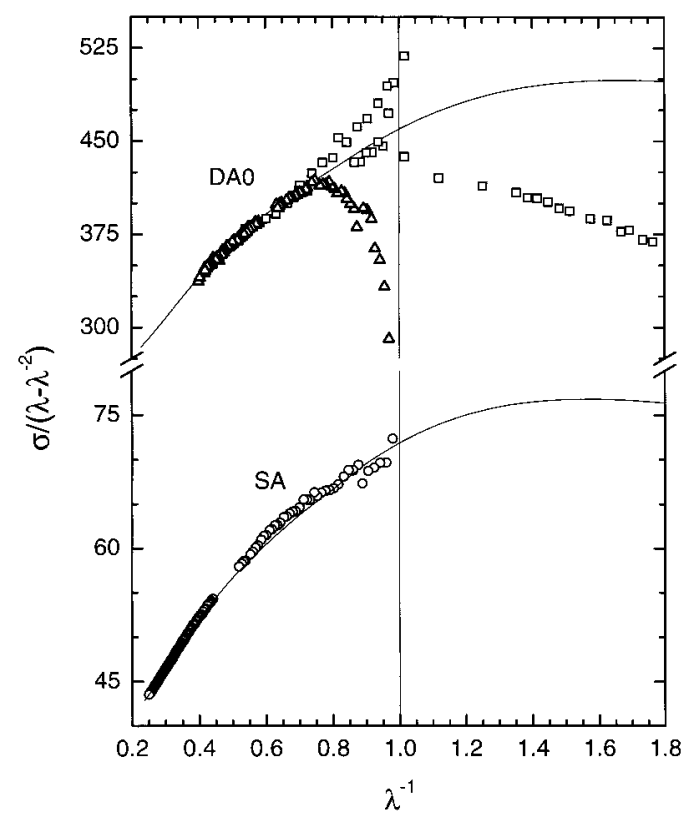

Figure 4. Reduced force versus inverse stretch ratio for two isotropic networks: cured once $(O)$ and twice $(\triangle)$. Also included are data ( $\square$ ) obtained earlier, ${ }^{32}$ on a network having the same composition as DAO. The solid line is a best fit of the DC theory, using only tension $\left(\lambda^{-1}<1\right)$ data. Note the break in the ordinate scale.

excellent agreement between DA0 and NR2, except for $\lambda$ near unity, where the experimental uncertainty approaches infinity. 32,54

The solid curves in Figure 4 represent the best fits to eq 6. It is known that the constraint theories of rubber elasticity cannot accurately describe experimental results involving more than one mode of deformation. ${ }^{30-32}$ Rather than poorly fit both the compression and tension data for DA0, we restrict the fit of the DC model to $\lambda^{-1}$ $<1$ (tension). This results in an overestimation of the stresses during compression. From these fits, we obtain $\mu_{1-2}=102 \mathrm{~mol} / \mathrm{m}^{3}$ and $\bar{\kappa}_{1-2}=3.0$ for DAO and $\mu_{1}=14.9$ $\mathrm{mol} / \mathrm{m}^{3}$ and $\bar{\kappa}_{1}=3.7$ for the precursor network SA. An inverse relationship between cross-link density and $\bar{\kappa}$ is in accord with theory 55 and with previous results, 56,57 including specifically peroxide-cured natural rubber networks. ${ }^{58}$

On the basis of the stability of the carbon-carbon covalent cross-links obtained by peroxide curing, ${ }^{59}$ we expect the first network to remain intact during the second cure; that is, $\mu_{1}$ retains the value of $14.9 \mathrm{~mol} /$ $\mathrm{m}^{3}$, and thus $\mu_{2}=\mu_{1-2}-\mu_{1}$. The effect of constraints on the first network, however, is not as obvious. If these constraints depend only on the cross-link density, the constraint parameter determined for the twice-cured network would characterize both components of the double networks, $\bar{\kappa}_{1-2}=\bar{\kappa}_{1}=\bar{\kappa}_{2}=3$. On the other hand, if the constraint for a given network is unchanged by the second curing, then $\bar{\kappa}_{1}=3.7$ and the value of $\bar{\kappa}_{2}$ is unknown. Fortunately, the results calculated for the double networks are relatively insensitive to the values of the two constraint parameters; herein we assume $\bar{\kappa}_{1}$ $=\bar{\kappa}_{2}=3$.

The analysis is based on the assumption that, for double networks at mechanical equilibrium and zero traction, the respective stresses from the component networks bal ance, i.e., $\sigma_{1}=-\sigma_{2}$ (eq 2). We can use eq 6 to predict the residual stretch $\lambda_{\mathrm{R}}$ at which this occurs, as governed by the cross-linking stretch $\lambda_{x}$. These

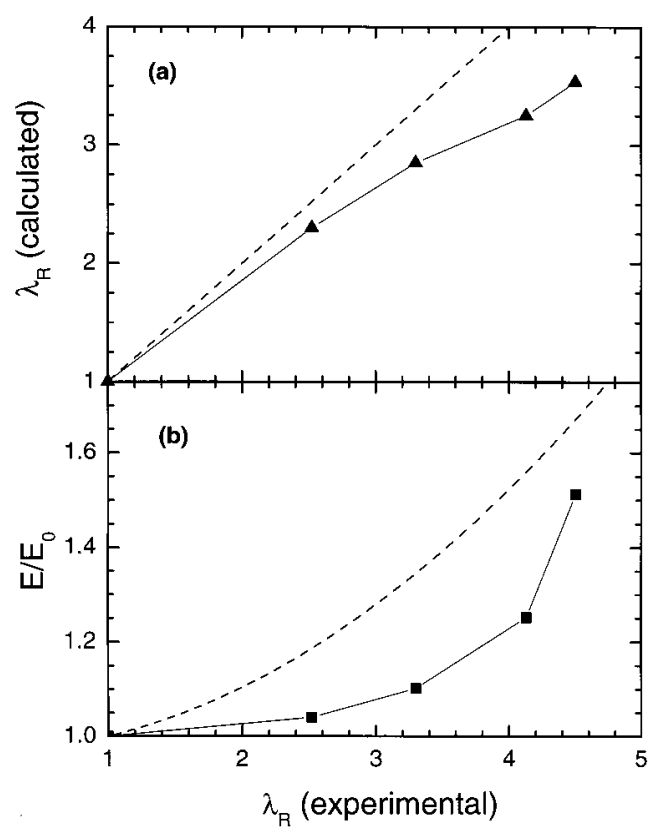

Figure 5. (a) Comparison of the residual stretch calculated using eqs 2 and $6(\mathbf{\Delta})$ to the experimental results $\left(45^{\circ}\right.$ dashed line), for networks of varying $\lambda_{x}$. (b) Comparison of the calculated modulus (dashed line) to the experimental results (a) expressed as $100 \%$ secant modulus, normalized by the value for the isotropic network having the same cross-link density.

calculated $\lambda_{R}$ are shown in Figure $5 a$ for the DA series double networks. In agreement with the experimental results, the calculated residual strain increases with increasing $\lambda_{x}$; however, as seen in Figure $5 a$, these calculated $\lambda_{\mathrm{R}}$ are less than the experimental values.

The possibility exists that degradation of the first network may reduce the retraction of the rubber following the second cure, thus contributing to a higher observed residual stretch. Indeed, as described above, oxidative degradation is sometimes observed at the sample edges. These were removed by trimming to produce uniform samples. We believe degradation plays a negligible role in the mechanical response of the double networks reported herein.

It is noteworthy that the residual strains are underpredicted, since this is consistent with the error in the rubber elasticity model. The calculated compressive stresses are too high (Figure 4), whereby it follows that the DC model will predict a higher retraction after the second cure, to balance the respective stresses of the component networks. The result that $\lambda_{\mathrm{R}}(\mathrm{calc})<\lambda_{\mathrm{R}}(\exp )$ can be taken as a confirmation of the finding of earlier work 31,32 on natural rubber networks, that the constraint models overestimate the stress in compression.

This limitation of the elasticity model means that we cannot expect more than qualitative predictions for double networks. Nevertheless, using eqs 1 and 6 , we calculate the stress of the double networks under deformation. These results are displayed in Figure $5 b$, which shows the $100 \%$ secant modulus, normalized by the modulus of the isotropic network having the same cross-link density, for various $\lambda_{R}$. With increasing residual strain, the predicted modulus increases, in the manner of the experimental results, although the agreement is not quantitative. For all experimental values of $\lambda_{R}$ herein, the modulus measured for the double network exceeds that of the corresponding isotropic network, although previous work found that this modu- 
Ius enhancement is not observed at lower residual strains $\left(\lambda_{R}<2\right) .^{5}$

Optical Birefringence. The birefringence of a single chain depends on the difference between its polarizability parallel and perpendicular to the deformation, 60

$$
\alpha_{x x}-\alpha_{y y}=\Gamma \frac{x^{2}-y^{2}}{\left\langle r^{2}\right\rangle}
$$

where $\Gamma$ represents the difference in bond polarizabilities along and transverse to the chain, $x$ and $y$ are the respective projections of the chain end-to-end vector along the two orthogonal directions, and $\left\langle r^{2}\right\rangle$ is the mean square average end-to-end vector. To obtain the macroscopic birefringence, $\Delta \mathrm{n}$, eq 14 is averaged over all chains. This gives ${ }^{60}$

$$
\overline{x^{2}}=\frac{1}{3} \lambda_{x}{ }^{2}\left\langle r^{2}\right\rangle, \quad \overline{y^{2}}=\frac{1}{3} \lambda_{y}{ }^{2}\left\langle r^{2}\right\rangle
$$

where $\lambda_{x}$ and $\lambda_{y}$ are the molecular extension ratios. F or uniaxial extension of an incompressible material, $\lambda=$ $\lambda_{\mathrm{x}}=\lambda_{\mathrm{y}}{ }^{-2}$, and the polarizability difference averaged over all chains is ${ }^{60}$

$$
\overline{\alpha_{x x}}-\overline{\alpha_{y y}}=\frac{\Gamma}{3}\left(\lambda-\lambda^{-2}\right)
$$

Obtaining an expression for the observed birefringence requires an elasticity model, to convert the molecular deformation into a macroscopic strain. In the classical rubber elasticity models, the difference between the two principal stresses is proportional to the righthand side of eq 16, yielding the stress optical law (eq 3). For real networks, the connection between the molecular extension and the macroscopic stress is more complicated. According to the DC model, ${ }^{61}$

$$
\begin{aligned}
& \Delta \mathrm{n}_{\mathrm{DC}}= \\
& \quad \Delta \mathrm{n}_{\mathrm{ph}}\left[1+2 \int_{0}^{1 \mathrm{~B}(\lambda)-\mathrm{B}\left(\lambda_{\perp}\right)+\mathrm{b}\left[\mathrm{D}(\lambda)-\mathrm{D}\left(\lambda_{\perp}\right)\right]} \frac{\lambda^{2}-\lambda^{-1}}{\lambda^{-1}} \mathrm{~d}\right]
\end{aligned}
$$

where $\Delta n_{p h}$, the birefringence of a phantom network, conforms to eq 3 . The parameter $b$, which lies between zero and unity, represents the direct contribution of the constraints to the birefringence. ${ }^{62}$ Equation 17 predicts deviation from a simple proportionality between optical birefringence and the true stress, as do the other constraint models. Such a breakdown of the stress optical law has been observed experimentally in mechanical equilibrium measurements on silicone ${ }^{63}$ and polybutadiene networks. ${ }^{64}$ It is also common during viscoelastic experiments on polymers. ${ }^{65-68}$

The strain dependence of the stress optical coefficient implicit in eq 17 can give rise to the zero-stress birefringence observed for the double networks. The basic hypothesis of double networks is that the stresses are additive. By analogy with eq 1, we express the measured birefringence as the sum of the contribution from the component networks

$$
\Delta \mathrm{n}_{1-2}=\Delta \mathrm{n}_{1}(\lambda)+\Delta \mathrm{n}_{2}\left(\lambda / \lambda_{\mathrm{x}}\right)
$$

In Figure 6 we plot the birefringence calculated from eq 17, for two values of the constraint parameter, $\bar{\kappa}$, with $b=1 / 2$. The data have been normalized by the true

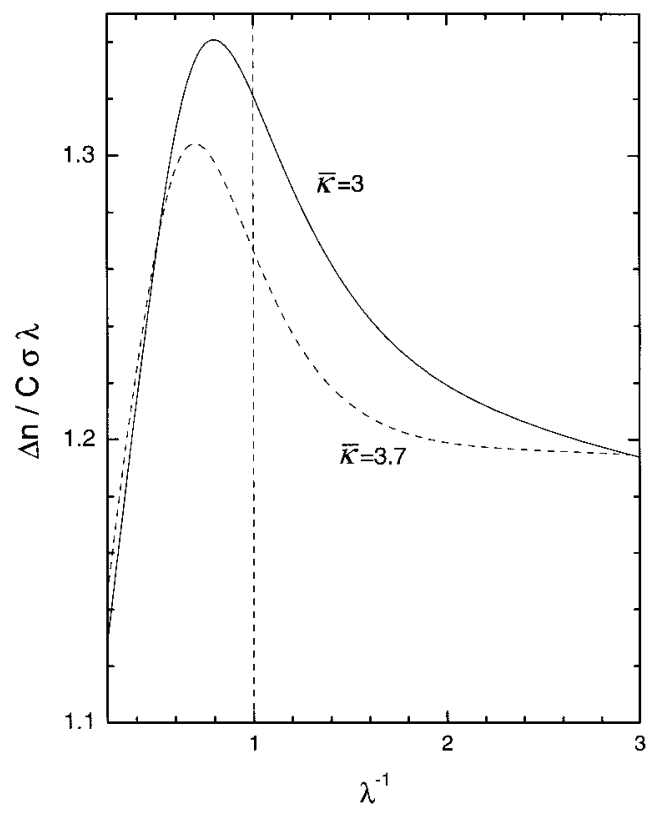

Figure 6. Normalized birefringence as a function of the inverse stretch ratio, as predicted by the constrained chain theory for the indicated values of $\bar{\kappa}$. Increasing $\bar{\kappa}$ from 3 to 3.7 displaces the peak toward larger extension, which can influence the birefringence of a double network rubber containing this component network.

stress, to indicate the deviation from the stress-optical law. There is an appreciable asymmetry, with the network constraints producing a larger birefringence for extension than in compression. To the extent eq 18 is valid, this provides a qualitative explanation for the data in Figure 2. The sign of $\Delta \mathrm{n}$ for the double networks, which corresponds to extension, is a consequence of the larger birefringence of the first network, relative to that of the second, at equal (absolute value of) true stress.

As described above, however, the DC model yields overly large compressive stresses. This makes any analysis of the birefringence data suspect, since a prediction of the zero-stress birefringence relies on the stress calculated for each component network. Additionally, while the assumption of independent network mechanical behavior is generally accepted, ,22-24 the further assumption of additivity of the respective birefringence from the two components (eq 18) may not be correct.

\section{Summary}

Stress, strain, and optical birefringence measurements on double networks provide a severe test of rubber elasticity models. The deficiency of the constrained chain model in fully describing the data herein is due at least in part to the fact that double network rubbers encompass both compression and tension. Nevertheless, while the theoretical treatment is not entirely satisfactory, the model does provide useful insight into the mechanical and optical behavior of double networks.

An obvious uncertainty in our analysis concerns the value of the constraint parameter for the component networks. In principle, one could fit stress-strain data for the double networks in order to deduce $\bar{\kappa}_{1}$ and $\bar{\kappa}_{2}$. This would reveal the effect of additional cross-linking and orientation on the constraints on the first network. Unfortunately, presently available elasticity models are inadequate to this task. 
The sign of $\Delta n_{1-2}$ measured herein corresponds to tension and in this regard is consistent with the birefringence calculated for the double networks. As seen from Figure 6 and eq 18, however, this sign is expected to be sensitive to the magnitude of $\bar{\kappa}$. It would be interesting to characterize the birefringence of other double network rubbers, such as polybutadiene, having a smaller entanglement molecular weight and higher junction functionality than peroxide-cured natural rubber. Calculations suggest this could occasion a change in the sign of the birefringence for unstressed double networks with $\lambda_{\mathrm{R}}>1$.

\section{References and Notes}

(1) Berry, J . P.; Scanlan, J .; Watson, W. F. Trans. Faraday Soc. 1956, 52, 1137.

(2) Scanlan, J.; Watson, W. F. Trans. Faraday Soc. 1958, 54, 740.

(3) Flory, P. J . Trans. Faraday Soc. 1960, 56, 722.

(4) Ullman, R. Macromolecules 1986, 19, 1748.

(5) Santangelo, P. G.; Roland, C. M. Rubber Chem. Technol. 1994 $67,359$.

(6) Santangelo, P. G.; Roland, C. M. Rubber Chem. Technol. 1995, 68, 124.

(7) Hamed, G. R.; Huang, M. Y. Rubber Chem. Technol. 1998, $71,846$.

(8) Kaang, S.; Nah, C. Polymer 1998, 39, 2209.

(9) Hvidt, S.; Kramer, O.; Batsberg, W.; Ferry, J . D. Macromolecules 1980, 13, 933.

(10) Batsberg, W.; Kramer, O. J . Chem. Phys. 1981, 74, 6507.

(11) Granick, S.; Ferry, J. D. Macromol ecules 1983, 16, 39.

(12) Kramer, O. ACS Symp. Ser. 1988, 367, 48.

(13) Twardowski, T. E.; Gaylord, R. J . Polym. Bull. 1989, 21, 393.

(14) Gaylord, R. J .; Twardowski, T. E.; Douglas, J. F. Polym. Bull. 1988, 20, 305

(15) Tobolsky, A. V.; Takahashi, Y.; Naganuma, S. Polym. J . 1972 3,60 .

(16) Gillen, K. T. Macromolecules 1988, 21, 442.

(17) Flory, P. J . J . Am. Chem. Soc. 1956, 78, 5222

(18) Mandelkern, L.; Roberts, D. E.; Diorio, A. F.; Posner, A. S. . Am. Chem. Soc. 1959, 81, 4148

(19) Hikmet, R. A. M.; Lub, J .; Vanderbrink, P. M. Macromolecules 1992, 25, 4194.

(20) Roland, C. M.; Peng, K. L. Rubber Chem. Technol. 1991, 64, 790.

(21) Reichert, W. F.; Goritz, D.; Duschl, E. J . Polymer 1993, 34, 1216.

(22) Andrews, R. D.; Tobolsky, A. V.; Hanson, E. E. J . Appl. Phys. 1946, 17, 352

(23) Baxandall, L. G.; Edwards, S. F. Macromolecules 1988, 21 , 1763.

(24) Termonia, T. Macromolecules 1990, 23, 1976.

(25) Greene, A.; Smith, K.J .; Ciferri, A. Trans. Faraday Soc. 1965, $61,2772$.

(26) Smith, K. J .; Gaylord, R. J . J . Polym. Sci., Part A-2 1972 10, 283.

(27) Pond, T. J .; Thomas, A. G. Proc. Int. Rubber Conf. (Venice, 1979), 810

(28) Roland, C. M.; Warzel, M. L. Rubber Chem. Technol. 1990, 63, 285.
(29) Riande, E.; Saiz, E. Dipole Moments and Birefringence of Polymers; Prentice Hall: Englewood Cliffs, NJ , 1992.

(30) Roland, C. M.; Mott, P. H.; Heinrich, G. Comput. Polym. Sci. 1999, 9, 197.

(31) Roland, C. M.; Mott, P. H. Macromolecules 1998, 31, 4033.

(32) Mott, P. H.; Roland, C. M. Macromolecules 1996, 29, 6941.

(33) Bennett, J . M. In Handbook of Optics, 2nd ed.; Bass, M., et al., Eds.; McGraw-Hill: New York, 1995; Vol. II.

(34) Yannas, I. V. J . Polym. Sci., Macromol. Rev. 1974, 9, 163.

(35) Larson, R. G. Constitutive Equations for Polymer Melts and Solution; Butterworth: Stoneham, MA, 1988; Chapter 3.

(36) Roland, C. M. Rubber Chem. Technol. 1989, 62, 880.

(37) Roland, C. M. J . Rheol. 1989, 33, 659.

(38) Saunders, D. W. Trans. Faraday Soc. 1956, 52, 1414.

(39) Mott, P. H.; Roland, C. M. Rubber Chem. Technol. 1995, 68, 739.

(40) Kloczkowski, A.; Mark, J. E.; Erman, B. Macromolecules 1995, 28, 5089.

(41) Ogden, R. W. Proc. R. Soc. London, A 1972, 326, 565.

(42) Valanis, K. C.; Landel, R. F. J . Appl. Phys. 1967, 38, 2997.

(43) Martin, G. M.; Roth, F. L.; Stiehler, R. D. Trans. Inst. Rubber Ind. 1956, 32, 189.

(44) Landel, R. F. Rubber Chem. Technol. 1998, 71, 234

(45) Arruda, E. M.; Boyce, M. C. J . Mech. Phys. Solids 1993, 41, 389.

(46) Arruda, E. M.; Przybylo, P. A. Polym. Eng. Sci. 1995, 35, 395.

(47) Gent, A. N. Rubber Chem. Technol. 1996, 69, 59.

(48) Boyce, M. C. Rubber Chem. Technol. 1996, 69, 781.

(49) Yeoh, O. H.; Fleming, P. D. J . Polym. Sci., Polym. Phys. 1997, 35, 1919.

(50) Flory, P. J . J . Chem. Phys. 1977, 66, 5720

(51) Flory, P. J .; Erman, B. Macromolecules 1982, 15, 800

(52) Erman, B.; Monnerie, L. Macromolecules 1989, 22, 3342.

(53) Coran, A. Y. Science and Technol ogy of Rubber; Mark, J . E., Erman, B., Eirich, F. R., Eds.; Academic Press: San Diego, 1994; Chapter 7.

(54) McKenna, G. B.; Zappas, L. J . Polymer 1983, 24, 1502.

(55) Pak, H.; Flory, P. J . J . Polym. Sci., Polym. Phys. Ed. 1979, $17,1845$.

(56) Brotzman, R. W.; Mark, J . E. Macromol ecules 1986, 19, 667.

(57) Hsu, Y.-H.; Mark, J . E.; Erman, B. J . Polym. Sci., Polym Phys. Ed. 1993, 31, 481.

(58) F ontaine, F.; Morland, C.; Noel, C.; Monnerie, L.; Erman, B. Macromolecules 1989, 22, 3348.

(59) Coran, A. Y. In Science and Technology of Rubber; Mark, J. E., Erman, B., Eirich, F. R., Eds.; Academic Press: New York, 1994; Chapter 7.

(60) Flory, P. J . Statistical Mechanics of Chain Molecules; Oxford University Press: New York, 1969.

(61) Kloczkowski, A.; Mark, J . E.; Erman, B. Comput. Polym. Sci. 1995, 5, 37

(62) Erman, B.; Flory, P. J . Macromolecules 1983, 16, 1601.

(63) Erman, B.; Flory, P. J . Macromolecules 1983, 16, 1607

(64) Stein, R. S.; Farris, R, J .; Kumar, S.; Soni, V. ACS Symp. Ser. 1982, 193, 453.

(65) Read, B. E. Polym. Eng. Sci. 1983, 23, 835.

(66) Osaki, K.; I noue, T.; H wang, E.-J .; Okamoto, H.; Takiguchi, O. J . Non-Cryst. Solids 1994, 172-174, 838.

(67) Mott, P. H.; Roland, C. M. Macromolecules 1998, 31, 7095.

(68) Roland, C. M.; Mott, P. H. Macromolecules 1999, 32, 4728. MA000145Q 